\title{
Renormalization Group Theory for Global Asymptotic Analysis
}

\author{
Lin-Yuan Chen, Nigel Goldenfeld, and Y. Oono \\ Department of Physics, Materials Research Laboratory, and \\ Beckman Institute, 1110 West Green Street, University of \\ Illinois at Urbana-Champaign, Urbana, IL 61801-3080, U. S. A.
}

\begin{abstract}
We show with several examples that renormalization group (RG) theory can be used to understand singular and reductive perturbation methods in a unified fashion. Amplitude equations describing slow motion dynamics in nonequilibrium phenomena are RG equations. The renormalized perturbation approach may be simpler to use than other approaches, because it does not require the use of asymptotic matching, and yields practically superior approximations.
\end{abstract}

Pacs numbers: 47.20.Ky, 02.30.Mv, 64.60.Ak 
The essence of the renormalization group (RG) is to extract those structurally stable features of a system which are insensitive to details. ${ }^{1,2}$ Thus, RG methods may be regarded as a means of asymptotic analysis. The usefulness of this point of view has been amply demonstrated ${ }^{3}$ by the relation between the RG and intermediate asymptotics, ${ }^{4}$ which showed that the anomalous exponents appearing in (e.g.) the long-time behavior of certain hydrodynamic systems were calculable using RG.

Many different techniques for asymptotic analysis have been developed including the multiple scaling (MS) method (which actually subsumes all the others), the boundary layer (BL) method, the WKB approximation, and others. ${ }^{5}$ Reductive perturbation methods ${ }^{6}$ have been used to extract the dynamics describing the global space-time behavior of complicated systems near bifurcation points. ${ }^{7}$

At a purely technical level, the starting point for both perturbative RG methods and conventional asymptotic methods is the removal of divergences from a perturbation series. Given the above similarities, a natural question arises: what is the relation, if any, between conventional asymptotic methods and the RG?

In this Letter, we demonstrate that many singular perturbation methods may be understood as renormalized perturbation theory, and that amplitude equations obtainable by the reductive perturbation methods are renormalization group equations. ${ }^{8}$ One of the advantages of the RG approach is that the starting point is simply a straightforward naive perturbation expansion, for which very little a priori knowledge is required; we will see that the RG approach seems to be more efficient and accurate, in practice, than standard methods in extracting global information from the perturbation expansion. 
To illustrate the basic idea, let us consider a weakly nonlinear van der Pol oscillator

$$
\frac{d^{2} y}{d t^{2}}+y=\epsilon\left\{\frac{d y}{d t}-\frac{1}{3}\left(\frac{d y}{d t}\right)^{3}\right\}
$$

which is usually solved by MS. The method of uniformization or 'renormalization' fails in this case. A naive expansion $y=y_{0}+\epsilon y_{1}+\epsilon^{2} y_{2}+\cdots$ gives

$$
\begin{aligned}
y(t)= & R_{0} \sin \left(t+\Theta_{0}\right)+\epsilon\left\{A_{1} R_{0} \sin \left(t+\Theta_{0}\right)+\left(B_{1}-\frac{R_{0}^{2}}{96}\right) R_{0} \cos \left(t+\Theta_{0}\right)\right. \\
& \left.+\frac{R_{0}}{2}\left(1-\frac{R_{0}^{2}}{4}\right)\left(t-t_{0}\right) \sin \left(t+\Theta_{0}\right)+\frac{R_{0}^{3}}{96} \cos 3\left(t+\Theta_{0}\right)\right\}+O\left[\epsilon^{2}\right],
\end{aligned}
$$

where $R_{0}, \Theta_{0}, A_{1}, B_{1}$ are constants determined by the initial conditions at arbitrary time $t=t_{0}$. This naive perturbation theory breaks down for $\epsilon\left(t-t_{0}\right)>1$ because of the secular terms. The arbitrary time $t_{0}$ may be interpreted as the (logarithm of the) ultraviolet cutoff in the usual field theory. ${ }^{3}$ To regularize the perturbation series, we introduce an arbitrary time $\tau$, split $t-t_{0}$ as $t-\tau+\tau-t_{0}$, and absorb the terms containing $\tau-t_{0}$ into the renormalized counterparts $R$ and $\Theta$ of $R_{0}$ and $\Theta_{0}$, respectively. This is allowed since $R_{0}$ and $\Theta_{0}$ are no longer constants of motion in the presence of the nonlinear perturbation. We introduce a multiplicative renormalization constant $Z_{1}=1+\sum_{1}^{\infty} a_{n} \epsilon^{n}$ and an additive one $Z_{2}=\sum_{1}^{\infty} b_{n} \epsilon^{n}$ such that $R_{0}\left(t_{0}\right)=Z_{1}\left(t_{0}, \tau\right) R(\tau)$ and $\Theta_{0}\left(t_{0}\right)=\Theta(\tau)+Z_{2}\left(t_{0}, \tau\right)$. The coefficients $a_{n}$ and $b_{n}(n \geq 1)$ are chosen order by order in $\epsilon$ to eliminate the terms containing $\tau-t_{0}$ as in the standard RG. The choice $a_{1}=-(1 / 2)\left(1-R^{2} / 4\right)\left(\tau-t_{0}\right)-A_{1}, b_{1}=-B_{1}$ removes the secular terms to order $\epsilon$, and we obtain the following renormalized perturbation result ${ }^{10}$

$$
\begin{aligned}
y(t)= & \left\{R+\epsilon(R / 2)\left(1-R^{2} / 4\right)(t-\tau)\right\} \sin (t+\Theta) \\
& -\epsilon(1 / 96) R^{3} \cos (t+\Theta)+\epsilon\left(R^{3} / 96\right) \cos 3(t+\Theta)+O\left[\epsilon^{2}\right],
\end{aligned}
$$


where $R, \Theta$ are now functions of $\tau$. Since $\tau$ does not appear in the original problem, the solution should not depend on $\tau$. Therefore, $(\partial y / \partial \tau)_{t}=0$. This is the RG equation, which in this case consists of two independent equations

$$
d R / d \tau=\epsilon(R / 2)\left(1-R^{2} / 4\right)+O\left[\epsilon^{2}\right], \quad d \Theta / d t=O\left[\epsilon^{2}\right]
$$

Setting $\tau=t$ in (3) eliminates the secular term, giving

$$
y(t)=R(t) \sin (t)+(\epsilon / 96) R(t)^{3} \cos (3 t)+O\left[\epsilon^{2}\right],
$$

where $R(t)$ and $\Theta(t)$ are obtained from (4) with $t=\tau: R(t)=R(0)\left[e^{-\epsilon t}+\right.$ $\left.R(0)^{2}\left(1-e^{-\epsilon t}\right) / 4\right]^{-1 / 2}$ and $\Theta(t)=\Theta(0)$, which we take to be zero. The final result approaches a limit circle of radius tending towards 2 as $t \rightarrow \infty$. The second order RG calculation shows that our assumption of perturbative renormalizability is consistent and no ambiguity arises. The multiple time scales used in MS for slow variables $T_{1}=\epsilon t, T_{2}=\epsilon^{2} t, \cdots$ appear naturally.

The above example illustrates two important points: (1) the results of the MS method can be obtained from renormalized perturbation theory, and (2) the RG equation describes the long time scale motion of the amplitude and the phase. In the following we wish to demonstrate (1) and (2) more generally.

Another important class of singular problems is that for which the highest order derivative of the equation is multiplied by a small parameter $\epsilon$, e.g. WKB and BL problems. For linear cases, it is known ${ }^{5}$ that both problems can be treated in a unified fashion. A typical problem is of the form

$$
\epsilon^{2} \frac{d^{2} y}{d x^{2}}+a(x) \frac{d y}{d x}-b(x) y=0, \quad 0 \leq x \leq 1, \quad \epsilon \rightarrow 0_{+},
$$

where $a$ and $b$ are continuous functions, and we have chosen $a(x)>0$ so that 
the boundary layer is at $x=0$. This can be transformed into a form suitable for WKB analysis:

$$
\epsilon^{2} \frac{d^{2} u}{d x^{2}}=Q(x) u(x)
$$

with $Q(x) \equiv a^{2}(x) / 4 \epsilon^{2}+a^{\prime}(x) / 2+b(x)$ and

$$
y(x)=\exp \left[-\frac{1}{2 \epsilon^{2}} \int^{x} a\left(x^{\prime}\right) d x^{\prime}\right] u(x)
$$

It is convenient to introduce a new variable $t$ such that $d t=\sqrt{Q} d x / \epsilon$, and (7) becomes

$$
\frac{d^{2} u}{d t^{2}}-u=-\frac{1}{2} Q^{-3 / 2}(x(t)) Q^{\prime}(x(t)) \frac{d u}{d t}
$$

where $Q^{\prime}(x(t))$ is assumed to be a function of order unity varying slowly on the time scale $t$, and $Q(x) \neq 0$ holds for $0 \leq x \leq 1$ for simplicity. Naively expanding $u$ as $u(t)=u_{0}(t)+\epsilon u_{1}(t)+\cdots$, we find the bare perturbation result

$$
\begin{aligned}
u(t)= & e^{t}\left\{A_{0}+\epsilon A_{0} \int_{t_{0}}^{t} S(x(s)) d s-\epsilon A_{0} e^{-2 t} \int_{t_{0}}^{t} S(x(s)) e^{2 s} d s\right\} \\
& +e^{-t}\left\{B_{0}+\epsilon B_{0} \int_{t_{0}}^{t} S(x(s)) d s-\epsilon B_{0} e^{2 t} \int_{t_{0}}^{t} S(x(s)) e^{-2 s} d s\right\}+O\left[\epsilon^{2}\right],
\end{aligned}
$$

where $S(x) \equiv-Q^{-3 / 2} Q^{\prime}(x) / 4$, and $A_{0}, B_{0}$ are constants dependent on the initial conditions at $t=t_{0}$. The terms in the curly brackets are the secular terms divergent in the limit $t-t_{0} \rightarrow \infty$. These terms are renormalized away with the aid of the multiplicative renormalization $A_{0}=Z_{1} A(\tau)$ and $B_{0}=Z_{2} B(\tau)$, where $A, B$ are the renormalized counterparts of $A_{0}, B_{0}$, respectively. Here $Z_{1}=$ 
$1+\epsilon \int_{\tau}^{t_{0}} S(x(s)) d s+\cdots=Z_{2}$, with $\tau$ being some arbitrary time, as in the example of the van der Pol oscillator. The renormalized perturbation result is

$$
u(t)=e^{t}\left\{A(\tau)+\epsilon A(\tau) \int_{\tau}^{t} S d s\right\}+e^{-t}\left\{B(\tau)+\epsilon B(\tau) \int_{\tau}^{t} S d s\right\}+O[\epsilon]
$$

where $O[\epsilon]$ refers to all regular terms of order $\epsilon$ which remain finite even as $t-t_{0} \rightarrow \infty$. The RG equation $\partial u / \partial \tau \equiv 0$ gives

$$
\frac{d C}{d \tau}+\epsilon \frac{1}{4} Q^{-3 / 2} Q^{\prime}(x(\tau)) C=O\left[\epsilon^{2}\right]
$$

where $C=A$ or $B$. Again, (12) corresponds to the amplitude equation with $\tau=t$, which gives $C(x) \sim Q^{-1 / 4}(x)$. This is just the adiabatic invariant $A(x) Q^{1 / 4}(x)=$ $A(0) Q^{1 / 4}(0)=$ constant. Therefore, the 'physical-optics' approximation for $(7)$ reads

$$
u(x) \sim Q^{-1 / 4}(x)\left\{C_{1} \exp \left[\frac{1}{\epsilon} \int^{x} d x^{\prime} \sqrt{Q\left(x^{\prime}\right)}\right]+C_{2} \exp \left[-\frac{1}{\epsilon} \int^{x} d x^{\prime} \sqrt{Q\left(x^{\prime}\right)}\right]\right\}
$$

where $C_{1}$ and $C_{2}$ are integration constants. The asymptotic result $y(x)$ uniformly valid for $\epsilon x<1$ for the general linear boundary-layer problem (6) is given by (8) with an appropriate asymptotic expansion formula for $Q(x)$. Notice that the above RG approach gives uniformly reliable results from the "inner expansion" alone without any (intermediate asymptotic) matching.

We have seen that the RG equation becomes the equation of motion for the slow behavior of the system. To see how RG reproduces reductive perturbation results, let us consider the following equation (this type covers most examples so 
far studied in the literature)

$$
\left[\mathcal{L}_{1}\left(\partial_{t}\right)+\mathcal{L}_{2}(\nabla)\right] u=\epsilon Q[u]
$$

where $\mathcal{L}_{1}$ and $\mathcal{L}_{2}$ are constant coefficient linear differential operators, $Q$ is a possibly nonlinear operator, and $\epsilon$ is a small parameter. We assume, for simplicity, spatial isotropy. Suppose that the operators have the following structures $\mathcal{L}_{1}=\prod_{\omega}\left(\partial_{t}+i \omega\right)^{m(\omega)}, \mathcal{L}_{2}=\prod_{\mu}(\nabla-i \mu)^{n(\mu)}$, and $u_{0}=\sum_{\omega, \mu} a_{\omega, \mu} e^{i(\mu \cdot x-\omega t)}$ is a solution to (14) with $\epsilon=0$. $^{12}$ The order $\epsilon$ correction, $u_{1}$, in the naive perturbation obeys $\left(\mathcal{L}_{1}+\mathcal{L}_{2}\right) u_{1}=Q\left[u_{0}\right]$. We assume without any loss of generality that $Q\left[u_{0}\right]$ can be expanded as

$$
Q\left[u_{0}\right]=\sum_{\omega, \mu} Q_{\omega, \mu}[\{a\}] e^{i(\mu \cdot x-\omega t)}+R
$$

where $R$ is the remainder such that $\mathcal{L}_{1} R \neq 0, \mathcal{L}_{2} R \neq 0$, and $Q_{\omega, \mu}$ are coefficients dependent on the set of $a_{\omega, \mu}$ 's collectively denoted by $\{a\}$. The general form of the singular (secular) part $\left[u_{1}\right]_{s}$ of the general solution is

$$
\begin{aligned}
{\left[u_{1}\right]_{s}=} & \sum_{\omega, \mu} Q_{\omega, \mu}[\{a\}]\left\{\lambda \ell_{1}(\omega)^{-1} \frac{t^{m(\omega)}-t_{0}^{m(\omega)}}{m(\omega) !}\right. \\
& \left.+(1-\lambda) \ell_{2}(\mu)^{-1} \frac{|x|^{n(\mu)}-\left|x_{0}\right|^{n(\mu)}}{n(\mu) !}+P_{\omega, \mu}\right\} e^{i(\mu \cdot x-\omega t)}
\end{aligned}
$$

where $\lambda$ is an arbitrary numerical constant, not equal to 0 or $1, P_{\omega, \mu}$ is a polynomial of $t$ and $|x|^{2}$ of lower order than $m(\omega)$ and $n(\mu) / 2$, respectively (whose explicit form is not required), $\ell_{1}(\omega)=\prod_{\omega^{\prime} \neq \omega}\left(i \omega-i \omega^{\prime}\right)^{m\left(\omega^{\prime}\right)}$ and $\ell_{2}(\mu)=$ $\prod_{\mu^{\prime} \neq \mu}\left(i \mu^{\prime}-i \mu\right)^{n\left(\mu^{\prime}\right)}$. Renormalization of the secular terms divergent in the global 
space-time limit can be done following the procedures given above, and is tantamount to replacing in (16) $t^{n}-t_{0}^{n}$ with $t^{n}-\tau^{n},|x|^{n}$ with $|x|^{n}-|r|^{n}$ and the 'bare' coefficients $\{a\}$ with their renormalized counterparts $\{A\}$, regarded as functions of $\tau$ and $r$. The renormalization group equation can be obtained from the condition that $u$ is independent of the parameters $\tau$ and $r$ introduced in the renormalization process. The term $P$ is dependent upon e.g., initial conditions. Thus, to obtain a universal result, we differentiate $u$ sufficiently many times with respect to $\tau$ and $r$ to eliminate $P$. Further eliminating $\lambda$, we find the following mode-coupled amplitude equation:

$$
\ell_{1}(\omega) \frac{\partial^{m(\omega)} A_{\omega, \mu}}{\partial t^{m(\omega)}}+\ell_{2}(\mu) \Delta^{n(\mu) / 2} A_{\omega, \mu}=\epsilon Q_{\omega, \mu}(\{A\}) .
$$

Here we have used the isotropy to introduce the Laplacian $\Delta$. This is the renormalization group equation independent of the arbitrariness due to initial conditions, solution methods, etc.

As an example, consider the Swift-Hohenberg equation: ${ }^{13}$

$$
\partial u / \partial t=\epsilon u-\left(1+\nabla^{2}\right)^{2} u-u^{3},
$$

where $\epsilon$ is a control parameter. Here $\mathcal{L}_{1}=\partial_{t}, \mathcal{L}_{2}=\left(1+\nabla^{2}\right)^{2}, \epsilon Q[u]=\epsilon u-u^{3}$ and $u_{0}=a e^{i x}+a^{*} e^{-i x}$. Thus $\ell_{1}=1, \ell_{2}( \pm 1)=-4, \epsilon Q_{0,1}\left(A, A^{*}\right)=\epsilon A-3|A|^{2} A$ and $\epsilon Q_{0,-1}\left(A, A^{*}\right)=\epsilon A^{*}-3|A|^{2} A^{*}$. That is,

$$
\frac{\partial A}{\partial t}=4 \frac{\partial^{2} A}{\partial x^{2}}+\left(\epsilon A-3|A|^{2} A\right) .
$$

Scaling out $\epsilon$ identifies the slow time and spatial variables as $T=\epsilon t, X=x / 2 \sqrt{\epsilon}$. The scaled result is the well-known time-dependent Ginzburg-Landau amplitude 
equation. Because the secular terms $\epsilon t$ and $\epsilon x^{2}$ have been removed from (16) the outcome (19) should be uniformly valid up to time scale $1 / \epsilon$ and spatial scale $1 / \sqrt{\epsilon}$ for $\epsilon \ll 1$. In our approach, spatial and time coordinates are treated on an equal footing, in contrast to the standard reductive perturbation method. 6

To demonstrate that there are not only conceptual but also technical advantages to the RG approach, we conclude with a problem involving the so-called 'switchback': conventionally, only through subtle analysis in the course of actually solving the problem is it possible to realize the need for (e.g.) unexpected order terms to make asymptotic matching consistent. An example is a caricature of the Stokes-Oseen singular boundary layer problem, which describes the low Reynolds number viscous flow past a sphere of unit radius. After scaling the radial coordinate $r$ to $x=\epsilon r$, where $\epsilon$ is the Reynolds number squared, the equation is ${ }^{14}$

$$
\frac{d^{2} u}{d x^{2}}+\frac{2}{x} \frac{d u}{d x}+u \frac{d u}{d x}=0, \quad u(x=\epsilon)=0, u(x=\infty)=1
$$

We regard (20) as an initial-value problem, given an initial condition $u\left(x_{0}\right)=A_{0}$ at some arbitrary point $x=x_{0}$, where $A_{0}$ is a finite constant. Assuming a naive expansion $u(x ; \epsilon)=u_{0}(x)+\lambda_{1}(\epsilon) u_{1}(x)+\lambda_{2}(\epsilon) u_{2}(x)+\cdots$, where $\lambda_{i}(\epsilon), i=1,2, \cdots$ will be determined in a self-consistent way, we obtain $u_{0}(x)=A_{0}$. Solving to $O\left[\lambda_{1}(\epsilon)\right]$ gives

$$
u(x)=A_{0}+\lambda_{1}(\epsilon) A_{1} A_{0}\left\{e_{2}\left(A_{0} x_{0}\right)-e_{2}\left(A_{0} x\right)\right\}+O\left[\lambda_{1}(\epsilon)^{2}, \lambda_{2}(\epsilon)\right]
$$

where $A_{1}\left(x_{0}\right)$ is some constant of integration, and we define $e_{2}(t) \equiv$ $\int_{t}^{\infty} d \rho \rho^{-2} e^{-\rho} \sim 1 / t+\log t+(\gamma-1)-t / 2+O\left(t^{2}\right)$ as $t \rightarrow 0$ with Euler's constant $\gamma \simeq 0.577$. The naive perturbation result (21) breaks down when both 
$x_{0}$ is small and $x-x_{0}$ is large. To cure this we introduce the renormalization constant $Z=1-\lambda(\epsilon) A_{1}\left\{e_{2}(A \mu)-e_{2}\left(A x_{0}\right)\right\}$ such that $A(\mu)=Z A_{0}$, giving the renormalized perturbation series

$$
u(x)=A(\mu)+\lambda_{1}(\epsilon) A_{1} A\left\{e_{2}(A \mu)-e_{2}(A x)\right\}+O\left[\lambda_{1}(\epsilon)^{2}, \lambda_{2}(\epsilon)\right]
$$

The RG equation $\partial u / \partial \mu=0$ yields, after setting $\mu=x$,

$$
\frac{d A(x)}{d x}=\lambda_{1}(\epsilon) A_{1} \frac{e^{-A(x) x}}{x^{2}}+O\left[\lambda_{1}(\epsilon)^{2}, \lambda_{2}(\epsilon)\right]
$$

and we find the final uniformly valid asymptotic result $u(x)=A(x)$.

Equation (23) can be solved iteratively along with the required boundary conditions $A(\infty)=1$ and $A(\epsilon)=0$ as

$$
A(x)=1-\lambda_{1}(\epsilon) A_{1} \int_{x}^{\infty} d \rho \rho^{-2} e^{-\rho}+O\left[\lambda_{1}(\epsilon)^{2}, \lambda_{2}(\epsilon)\right] .
$$

The condition $A(\epsilon)=0$ gives $\lambda_{1}(\epsilon) A_{1} e_{2}(\epsilon)=1$. Therefore, the expansion coefficient $\lambda_{1}$ can be explicitly chosen as $\lambda_{1}(\epsilon) A_{1}=1 / e_{2}(\epsilon)$, with the asymptotic expansion, in the limit $\epsilon \rightarrow 0_{+}, A_{1} \lambda_{1}(\epsilon) \sim \epsilon(1-\epsilon \log \epsilon-(\gamma-1) \epsilon+\cdots)$. In addition, we require that $\lambda_{1}^{2} / \lambda_{2}=O(1)$, so that the equation for $u_{2}$ yields new information. The resulting asymptotic solution is correct to $O[\epsilon \log \epsilon]$ and agrees with that obtained by asymptotic matching. Note that in our method, the $\epsilon \log \epsilon$ term appears naturally from the asymptotic expansion of $e_{2}(\epsilon)$, whereas some artistry is required to obtain this term conventionally. The result to $O[\epsilon]$ given by asymptotic matching ${ }^{14}$ is obtained from the renormalized perturbation expansion to $O\left[\lambda_{2}\right]$. The asymptotic expansion to $O[\epsilon]$ is not uniformly valid in $r$, and a much better approximation, in practice, is our full result $1-e_{2}(\epsilon r) / e_{2}(\epsilon)$ to order $\lambda_{1}$. This can be seen clearly in figure 1 . 
In summary, we have demonstrated that various singular perturbation methods and reductive perturbation methods may be understood in a unified fashion from the renormalization group point of view, with some attendant technical advantages.

We are grateful to Paul Newton for valuable discussions. LYC acknowledges the support of grant NSF-DMR-89-20538, administered through the University of Illinois Materials Research Laboratory. NG and YO gratefully acknowledge partial support by National Science Foundation Grant NSF-DMR-93-14938.

\section{REFERENCES}

1. N. D. Goldenfeld, Lectures on Phase Transitions and the Renormalization Group (Addison-Wesley, Reading, Mass., 1992).

2. L. Y. Chen, N. Goldenfeld, Y. Oono and G. Paquette, Physica A 204, 111 (1994); G. Paquette, L. Y. Chen, N. Goldenfeld, and Y. Oono, Phys. Rev. Lett. 72, 76 (1994).

3. N. Goldenfeld, O. Martin and Y. Oono, J. Sci. Comp. 4, 355 (1989); N. Goldenfeld, O. Martin, Y. Oono and F. Liu, Phys. Rev. Lett. 64, 1361 (1990); J. Bricmont and A. Kupiainen, Commun. Math. Phys. 150, 193 (1992).

4. G. I. Barenblatt, Similarity, Self-Similarity, and Intermediate Asymptotics (Consultant Bureau, New York, 1979).

5. For example, C. M. Bender and S. A. Orszag, Advanced Mathematical Methods for Scientists and Engineers (McGraw-Hill,1978); J. Kevorkian and J. D. Cole, Perturbation Methods in Applied Mathematics (Springer, New York, 1981). 
6. T. Taniuti and C. C. Wei, J. Phys. Soc. Jpn. 24, 941 (1968); A. C. Newell and J. A. Whitehead, J. Fluid Mech. 38, 279 (1969); Y. Kuramoto, Chemical Oscillations, Waves, and Turbulence (Springer, Berlin, 1984).

7. See, e.g., J.D. Crawford, Rev. Mod. Phys. 63, 991 (1991); M.C. Cross and P.C. Hohenberg, Rev. Mod. Phys. 65, 851 (1993).

8. We emphasize that our RG method has no connection with the so-called method of renormalization or uniformization, which is a variant of the method of stretched coordinates, and of limited use.

9. E. C. G. Stueckelberg and A. Petermann, Helv. Phys. Acta 26, 499 (1953); M. Gell-Mann and F. E. Low, Phys. Rev. 95, 1300 (1954); J. Zinn-Justin, Quantum Field Theory and Critical Phenomena (Clarendon Press, Oxford, 1989).

10. $Z_{1}$ may depend upon $R$, because $R$ is dimensionless. This is analogous to the renormalization of a dimensionless coupling constant in field theory.

11. A related result has been obtained independently by S.L. Woodruff, Studies in Applied Mathematics 90, 225 (1993).

12. Those $\mu$ and $\omega$ which are not real do not give a bounded, globally meaningful zeroth order solution, so they must be excluded from the expression for $u_{0}$.

13. J. Swift and P. C. Hohenberg, Phys. Rev. A 15, 319 (1977).

14. E. J. Hinch, Perturbation Methods (Cambridge University Press, Cambridge, 1991), section 5.2. 


\section{FIGURE CAPTIONS}

Fig. 1. Comparison between the numerical solution of eq. (20) for several values of $\epsilon$, the first order RG result $1-e_{2}(\epsilon r) / e_{2}(\epsilon)$, and two matched asymptotic expansions (one at fixed $r$, the other at fixed $\rho \equiv r \epsilon$ ), as derived in ref. 14 . 